\title{
Throughput analysis in an automated material handling system
}

\author{
P. Simin Pulat \\ Assistant Professor \\ Department of Industrial Engineering \\ University of Oklahoma \\ Norman, OK 73019
}

\author{
B. Mustafa Pulat \\ Senior Engineer \\ AT\&T Technologies, Inc. \\ Oklahoma City Works \\ Oklahoma City, OK 73126-0060
}

P. SIMIN PULAT is an assistant professor in industrial engineering at the University of Oklahoma. She received her MS and Ph.D. degrees in operations research at North Carolina State University. Her research interests include optimization, network analysis, network reliability and maintainability, multicriteria decision making and stochastic modeling.

B. MUSTAFA PULAT is a senior engineer in the material handling engineering department of AT\&T Oklahoma City Works. His degrees are all in industrial engineering including a Ph.D. from North Carolina State University in Raleigh, NC. Mustafa published extensively in the areas of ergonomics, modeling and computer integrated manufacturing including Industrial Ergonomics: A Practitioner's Guide published by IIE in 1985. At AT\&T, Mustafa carries out systems engineering and automation projects in material and information movement and management.

\begin{abstract}
Throughput analysis in an automated material handling system, such as an Automated Storage and Retrieval System(AS/RS), may be a complex problem. In the past, several approaches have been used for such an analysis. This paper presents a combinatorial approach to evaluating the throughput performance of a mini-load system, with simulation as the primary method of investigation.
\end{abstract}

\section{INTRODUCTION}

Efficient material handling is one of industry's most challenging problems. This is especially evident in the electrical and electronics manufacturing industry where material and handling costs typically average around $60-80 \%$ of product cost.

Nowadays, many material handling functions are being automated. Furthermore, in order to reduce inventory investment, many industries are implementing JIT(Just-In-Time) programs. However, not every part may be handled on a just-in-time basis. With a $20 \%$ IIT proportion, a factory handling 50,000 parts faces the problem of effectively buffering 40,000 parts between receiving and manufacturing. This frequently calls for automated handling.

Among several technologies developed for efficient material handling in receiving, buffering and dispatching functions, the most ad- vanced is probably an Automated Storage and Retrieval System(AS/RS). The AS/RS technology was developed in the 1960s with advances in real time control of machines by computers. AS/RSs are highly specialized systems which require considerable capital investment. Their speed, accuracy, and capacity must be adequate to justify the expenditures.

\section{PROBLEM STATEMENT}

Oklahoma City Works of AT\&T Technologies operationalized a mini-load AS/RS two years ago. The system displays 48,000 storage adresses in a nine aisle structure with 35 bays and 45 rows. Built to seismic specifications, the structure supports four bin sizes. Aisle captive S/R(storage and retrieval) cranes(robots) are responsible for delivering bins to $\mathrm{l} / \mathrm{O}$ (input/output) points. The AS/RS is responsible for receiving, buffering, selecting(picking), and auditing(counting) of primarily circuit pack components.

Prior to the development of the system, several studies established the expected transaction capacity in terms of number of selects, audits, and stocking activities per shift. The first few Material Requirements Planning(MRP) logical stores were loaded on the system on the basis of the results of these studies. However, before proceeding any further, another and more comprehensive mechanical and controlling software capability study with real performance data was carried out. This paper presents the details of the mechanical handling capacity studies.

\section{AS/RS MATERIAL FLOWS}

The AS/RS receives material from several sources(see Figure 1) including the receiving dock, the Integrated Circuit Test and Firmware shop, other shops and buffer zones, and areas within its control(accumulator aisles). At the receiving stations, material is either routed to stocking or shortage fill area or both. Material to be buffered is stocked into bins delivered to the input point by a crane. Bins are then taken back to their assigned locations in the rack structure. Material routed to the shortage fill area is used to fill short selects and/or floor shortages. 


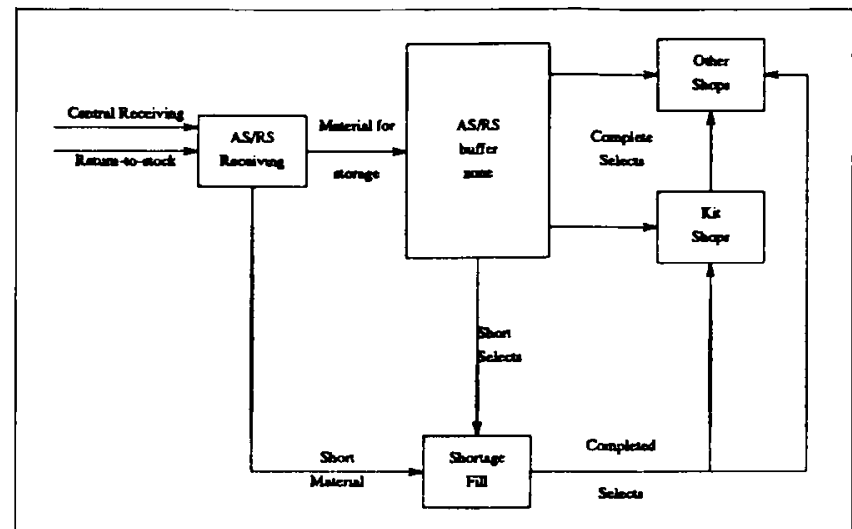

Figure 1. AS/RS Material Flow.

Selecting and inventory audits are accomplished at the P/D(pick and delivery) stations. Complete selects are moved to the kit shops or other manufacturing shops. Short selects are staged until filled. They are then moved to the point-of-use.

\section{PICK AND DELIVERY(P/D) STATIONS}

There are two $\mathrm{I} / \mathrm{O}$ points for each crane. The stocking station is at the mezzanine level. Picking and inventory audit functions are carried out at the P/D station at the floor level. Most of the system activity is due to pick and inventory audit $(88 \%)$. Thus it is important that the hardware and work flow at each P/D be reviewed.

\subsection{P/D Hardware and Work Flow}

Material flows in a horseshoe pattern through each station. Inbound bins are queued on an elevated roller conveyor. An elevator lowers the next bin in the work queue down to the operator level. The bin on which work has been completed is lowered to an outbound bins queue for crane pickup. The inbound bin queue length is controlled via photoelectric cells. When the queue length reaches five, bin delivery to that station stops. The process resumes with four or less bins in the queue. The outbound bin queue length limit is three. When this happens, the operator is blocked since no more bins can be processed. Each crane serves two P/D stations.

\section{METHODOLOGY}

Since the performance of the system largely depends on the activity level at the $P / D$, investigations concentrated on the output per $P / D$ pair in terms of number of bins processed and load completion times. However, linearity assumption between one aisle's output and system output had to be justified with checks on level loadedness of aisles in terms of number of parts and their frequency of use, and long term randomization of load distribution between aisles. All checks proved that these assumptions were valid.

Several approaches have been utilized in the past to carry out AS/RS throughput analyses. The analytic approach concentrated on developing mathematical models to approximate system output. Chow(1983), Bozer(1986), and McCinnis and Trevino(1985) may provide some examples. The simulation approach was used for the same purpose, relaxing the requirement for mathematical relationships. Bafna(1972), Han(1984), and Rizo-Patron et al.(1983) are sample studies. The work measurement approach concentrates on output observations per unit time, and projections to system output usually in a shift. Any classic work measurement text will provide examples(see Niebel 1982, and Barnes 1980).

Three simultaneous investigations were carried out for process characterization in this study. Simulation was the primary tool utilized. In addition, a queueing theoretic approach and intensive sampling of operator activity were employed. The sampling study was performed in order to cross check the results of the first two analysis methods.

\subsection{Computer Simulation}

A SIMAN model(see Pegden 1984) served as the simulation tool(see Figures 2\&3). In Figure 2, entities representing bins in quantity equal to an average daily load are generated as the first step and placed in a queue. This is realistic since MRP download of daily work occurs each night in a batch mode. Each entity is assigned an attribute code representing the type of activity to be performed (select or audit) on the bin. $A$ bin is delivered to the $P / D$ inbound bins queue only if there exists four or less entities in the queue.

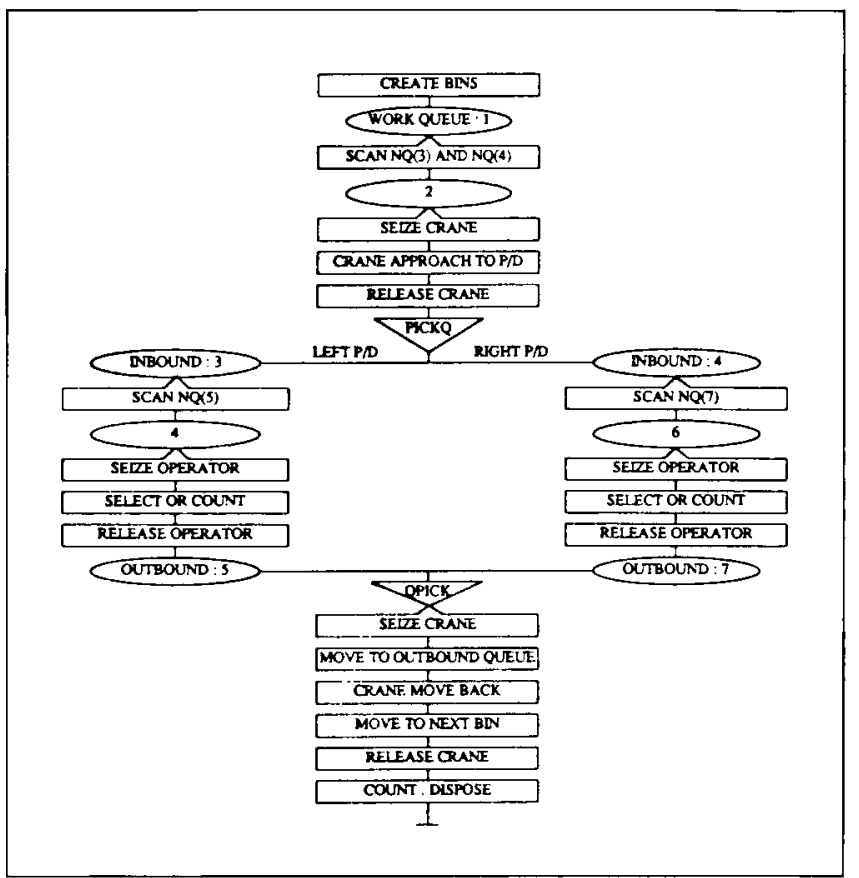

Figure 2. Siman Model of the P/D Process.

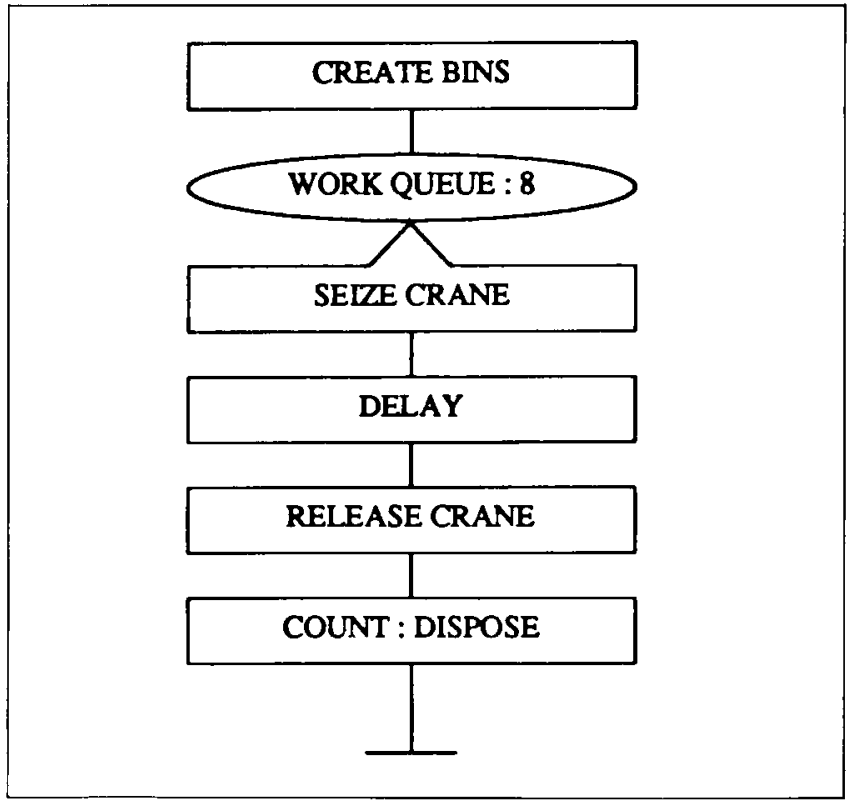

Figure 3. Siman Model of the Stocking Process. 
Bins in the inbound queue are processed one at a time by the operator only if the system is not blocked. If so, the operator spends time in interference idleness. Each bin is processed according to the work type. This process is the same for both the left and the right $P / D$. Duty cycle time is drawn from an appropriate discrete probability distribution. In the mean time, the crane is performing dual command cycles.

Subsequent to bin delivery to the inbound queue, if there is a bin in the outbound queue, the crane is scheduled to move down to pick it and deliver to its assigned rack location. Then the crane moves to the rack location where the next bin to be processed resides. A counter is incremented after returning a bin to its location and the entity is disposed.

Figure 3 gives a similar crane schedule for stocking activity. In both figures 2 and 3, activity sequence end when all entities are processed. Simulation ends when the last $P / D$ or stocking bin is processed.

All crane move times are drawn from appropriate discrete probability distributions. The discrete probability distributions utilized were empirically developed.

\subsection{Queueing Theory Model}

The queueing theoretic approach(see Gross and Harris 1974) is described using Figure 4.

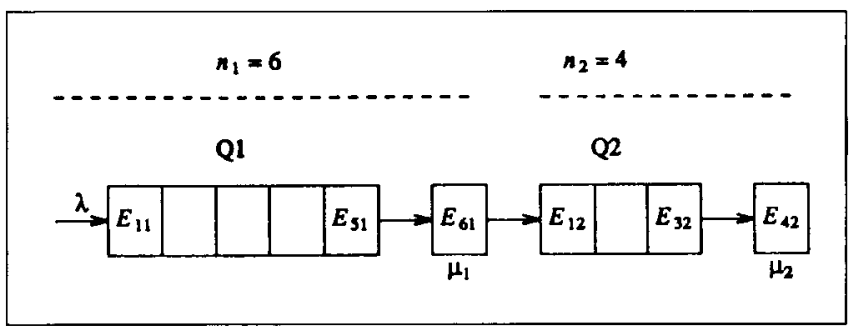

Figure 4. The P/D Process as a Queueing System

Consider a tandem network involving two stations. Each station has a single server and system capacity of size $n_{1}$ and $n_{2}$. The first station is represented by the inbound bins queue coupled with the operator's work area. The second station is represented by the outbound bins queue and the crane pickup. In Figure $4, n_{1}=6$ (queue length $=5$ ) and $n_{2}=4$ (queue length $=3$ ). If station two is full and service is completed at station one, the customer(a bin) must wait until service is completed at station two. During this time the server ( $P / D$ operator) in station one remains idle. This case is referred to as station one being blocked. Arrivals at station one are turned away (crane not delivering) when the station is full. Arrivals to station one are assumed to be Poisson distributed with parameter $\lambda$ and service times are exponential with parameters $\mu_{1}$ and $\mu_{2}$, respectively. The states of the system are defined in Table 1. The state definition indicates that there are $\left(n_{1}+1\right) \times\left(n_{2}+1\right)$ states where station one is not blocked and $n_{1}$ states where it is blocked. 41 states exist for our problem. We wish to compute the steady state probabilities $P_{i, 0, k}$ and $P_{i, b, n_{2}}$ for all $i$ and k. Specifically, we are interested in $P_{6,0,0}$ which represents crane idleness, and the total blockage probability $P_{b}$ given by:

$$
P_{b}=\sum_{i=1}^{6} P_{i, b, 4}
$$

Let $i$ and $k$ denote the number of customers in stations 1 and 2, respectively.
Table 1. System States

\begin{tabular}{|c|c|c|}
\hline State & Description & Limits on Subscripts \\
\hline$i, 0, k$ & $\begin{array}{c}i \text { customers in station 1 } \\
\text { station 1 not blocked } \\
k \text { customers in station 2 }\end{array}$ & $i=0, \ldots \ldots, n_{1}$ \\
$i, b, n_{2}$ & $\begin{array}{c}i \text { customers in station 1 } \\
\text { station 1 blocked } \\
n_{2} \text { customers in station 2 }\end{array}$ & $i=0, \ldots \ldots, n_{2}$ \\
\hline
\end{tabular}

Define,

$$
\begin{gathered}
\lambda_{i}= \begin{cases}\lambda & 0 \leqslant i<6 \\
0 & \text { otherwise }\end{cases} \\
\mu_{1 i}= \begin{cases}\mu_{1} & 0<i \leqslant 6 \\
0 & \text { otherwise }\end{cases}
\end{gathered}
$$

and,

$$
\mu_{2 k}=\begin{array}{cl}
\mu_{2} & 0<k \leqslant 4 \\
0 & \text { otherwise }
\end{array}
$$

Steady state equations can then be written as follows:

$$
\lambda_{0} P_{0,0,0}=\mu_{21} P_{0,0,1}
$$

$$
\begin{aligned}
& \left(\lambda_{0}+\mu_{2 k}\right) P_{0,0, k}=\mu_{11} P_{1,0,(k-1)}+\mu_{2(k+1)} P_{0,0,(k+1)} \text { for } k=1, \ldots, n_{2}-1 \\
& \left(\lambda_{i}=\mu_{1 i}\right) P_{i, 0,0}=\lambda_{(i-1)} P_{(i-1), 0,0}+\mu_{21} P_{i, 0,1} \text { for } i=1, \ldots . ., n_{1} \\
& \left(\lambda_{i}+\mu_{1 i}+\mu_{2 k}\right) P_{1,0, k}=\lambda_{(i-1)} P_{(i-1), 0, k}+\mu_{1(i+1), 0,(k-1)}+\mu_{2(k+1)} P_{i, 0,(k+1)} \\
& \text { for } i=1, \ldots, n_{1} \\
& \text { for } j=1, \ldots, n_{2}-1 \\
& \left(\lambda_{0}+\mu_{2 n_{2}}\right) P_{0,0, n_{2}}=\mu_{11} P_{1,0,\left(n_{2}-1\right)}+\mu_{2 n_{2}} P_{1, b, n_{2}} \\
& \left(\lambda_{i}+\mu_{1 i}+\mu_{2 n_{2}}\right) P_{i, 0, n_{2}}=\lambda_{(i-1)} P_{(i-1), 0, n_{2}}+\mu_{1(i+1)} P_{(i+1), 0,\left(n_{2}-1\right)}+\mu_{2 n_{2}} P_{(i+1), b, n_{2}} \\
& \text { for } i=1, \ldots, n_{1}
\end{aligned}
$$

and,

$$
\left(\lambda_{i}+\mu_{2 n_{2}}\right) P_{i, b, n_{2}}=\lambda_{(i-1)} P_{(i-1), b, n_{2}}+\mu_{1 i} P_{i, 0, n_{2}} \text { for } i=1, \ldots, n_{1}
$$

The above system of equations are linearly dependent. Any one of the equations can be eliminated while solving for the unknowns. There exists $\left(n_{1}+1\right) \times\left(n_{2}+1\right)+n_{1}-1$ equations and $\left(n_{1}+1\right) \times\left(n_{2}+1\right)+n_{1}$ unknowns. One can uniquely determine the steady state probabilities with the following additional equation:

$$
\sum_{i=0}^{n_{1}} \sum_{k=0}^{n_{2}} P_{0, i, k}+\sum_{i=1}^{n_{1}} P_{i, b_{,}, n_{2}}=1
$$

The performance analysis of the model and comparison with the other approaches are discussed in section 6. 


\subsection{Intensive Sampling}

This leg of the reseach was conducted to validate the simulation model and cross check the queueing results. A completely randomized intensive activity sample was carried out within operational blocks by three different members of the material handling engineering department. The operational blocks in question are shifts(day and evening), P/D stations(one through eighteen), and finally four hour time blocks within a shift(first versus second). All this effort was to account for possible sources of variation which may affect the percent time spent at various operating status by the operators. Fourteen such conditions were tracked, the most important being productive, blocked, starved and avoidable delays.

\section{RESULTS}

Although the intensive sampling approach evaluated system status in various operating conditions, two are most important for system performance comparison purposes across the research tools. These are:

1. Operator interference idleness(system blocked).

2. Crane idleness.

In the optimal design of the system, neither should have a nonzero value. However, within the real life constraints of a manufacturing environment, one will. Table 2 gives the comparative results of the investigation across the two performance measures.

Table 2. Results (in percentages).

\begin{tabular}{|l|c|ccc|}
\hline & & \multicolumn{3}{|c|}{ Analysis Technique } \\
\cline { 3 - 5 } \multicolumn{1}{c|}{$\begin{array}{c}\text { Performance } \\
\text { Measures }\end{array}$} & Operation & \multicolumn{4}{|c|}{ Queueing } & Intensive \\
Type & Simulation & Theory & Sampling \\
\hline \hline Operator Interference & Select & 3 & 21.5 & 4 \\
Idleness & Audit & 41 & 33 & 55 \\
(System Blocked) & Mix & 27 & 25.3 & 24 \\
\hline \hline & Select & 0 & 0.02 & 0 \\
Crane Idleness & Audit & 0 & 0.002 & 0 \\
& Mix & 0 & 0.01 & 0 \\
\hline
\end{tabular}

As can be observed, the bottleneck operation is crane handling of bins. This is evident in zero expected idleness(except for breakdown) on this resource. Thus, system output is determined by crane performance, including speed and mechanical availability. Operators on the other hand display various degrees of interference idleness depending on the P/D operation type and analysis technique. Most idleness occurs when both sides of the $P / D$ are scheduled with bin audits. This is a consistent result obtained across all approaches employed. It is a plausible outcome since when bin audits are scheduled for the P/Ds, operators can process bins at a much higher rate than the crane can process bins. Thus, the outbound queue will be filled fast and station one will be blocked most of the time.

Another consistent result across the analysis techniques is that the operators spend the second most time in interference idleness when a mix of selects and audits is scheduled for the P/Ds. In this case, aggregate operator bin processing rate gets closer to the crane bin processing rate. Least interference idleness occurs when all selects are scheduled. This is again a consistent result across all research tools, which can be explained by the fact that in this case, the processing rate difference between the two resources is minimum.

Although consistent trends have been observed in terms of results between the approaches, one can also observe differences in numeric results between tools within a given performance measure. An example is that percent operator interference idleness ranges between 3 and 22 percent for selects across the research tools employed. Some of these differences may be attributed to the fact that the queueing approach assumes exponential arrival and service times. This assumption may not hold since the AS/RS administrator monitors and adjusts work mix and priorities throughout the day. Intensive sampling data have been collected over a considerable period, but may not have captured all sources of variation.

Furthermore, event classification in terms of P/D operation type can not be made in clear terms with a sufficient number of data points. However, simulation data has been developed over a week's period across both shifts and several P/Ds via two independent observers. Nevertheless, there is more agreement between simulation and intensive sampling results than there is between queueing theory and intensive sampling results. Thus, we have more confidence in the simulation results. Hence, the system capacity decisions in terms of number of bin pulls per shift have been based on the simulation results. Consistent trends in the behavior of the performance measures across the investigation tools, and agreement between simulation and intensive sampling results give simulation the needed feel for predictive validity.

\section{CONCLUSIONS}

It is evident that computer simulation can be effectively utilized for AS/RS performance analysis. Throughput capacity may be determined by modeling operator and crane activity at the P/D with allowance for stocking station service as displayed by this study.

Simulation is also an effective tool for identifying bottleneck operation which determines the system output capacity. The AS/RS crane mechanical uptime percentage is a variable that is very closely monitored at the Oklahoma City Works. The AS/RS has a dedicated maintenance crew with software capabilities, including a preexpert system for trouble-shooting.

Evaluation of alternate AS/RS design parameters may also be carried out through computer simulation. Given a transaction load per unit time(week, day, shift), one may determine critical design parameters such as number of aisles, bays and rows, number of $\mathrm{I} / \mathrm{O}$ points and their locations, and P/D design parameters. This approach was implemented during planning for additional automation in the manual storeroom zones at the Oklahoma City Works.

\section{REFERENCES}

Bafna, K. M. 1972. Procedures Given for Determining AS/RS System Size and Preparing Specs., Industrial Engineering, (August): 76-81.

Barnes, R. M. 1980. Motion and Time Study (7'th Ed.), John Wiley and Sons, New York, NY

Bozer, Y. A. 1986. Optimizing Throughput Performance in Designing Order Picking Systems, Technical Report MHRC-TD-86-07. Material Handling Research Institute, Georgia Institute of Technology, Atlanta GA.

Chow, W. 1983. An Analysis of Automated Storage and Retrieval Systems in Manufacturing Assembly Lines, IBM Technical Report TR 02.1082, IBM Ceneral Products Division, San Jose, CA.

Gross, D. and Harris, C. M. 1974. Fundamentals of Queueing Theory, John Wiley and Sons, New York, NY.

Han, M. 1984. Evaluation of Crane Dispatching Rules, Presented at the Dallas ORSAVIIMS Conference, Dallas TX

McGinnis, L. F. and Trevino, J. 1985. Integrated Analysis of Nonsynchronous Assembly Systems Used in the Electronic Industry, Presented at the Intemational Assembly Conference, Santa Clara, CA.

Niebel, B. W. 1982. Motion and Time Study ( 7 'th Ed.), Richand Irwin, Homewood, IL. Pegden, D. C. 1984. Introduction to SIMAN, Systems Modeling Corporation, State College, PA.

Rizo-Patron, A., Bozer, Y.A., and MCGinnis, L.F. 1983. Analytic and Simulation Models for Advanced Automated Storage/Retrieval Systems, Technical Report TR-82-03. Material Handling Research Center, Georgia Institute of Technology, Atlanta, GA 